\title{
Unicellular Eukaryote as a Bio-cellular Model for Studying Effect Benzimidazole: Ultrastructural Analysis
}

\author{
Hovnanyan $\mathrm{KO}^{1 *}$, Gasparyan $\mathrm{HV}^{1}$, Hovnanyan $\mathrm{MK}^{1}$ and Trchounian $\mathrm{AA}^{2}$ \\ ${ }^{1}$ Center of Technological Organic and Pharmaceutical Chemistry of NAS RA, Armenia \\ ${ }^{2}$ Department of Biochemistry, Microbiology and Biotechnology, Yerevan State University, \\ Armenia
}

*Corresponding author: Karlen O Hovnanyan, Center of Technological Organic and Pharmaceutical Chemistry of NAS RA, 26 Azatutyan Str. 0014, Yerevan, 0014, Armenia, Email: hovkarl@mail.ru

\section{Research Article}

Volume 4 Issue 1

Received Date: December 31, 2020

Published Date: January 22, 2021

DOI: $10.23880 /$ oajmms-16000132

\section{Abstract}

The search for new derivatives of benzoimidazole with an active center that have a wide range of biological effects (antifungal, antiflammatory, antibacterial, antiviral, antitumor, antidiabetic, etc.) is the subject of modern pharmaceutical science.

To determine the mechanism of action of benzoimidazole, a search is underway for more. We have previously carried out the Ultrastructural characterization of various prokaryotes and protists, as well as the mechanism of action of antibiotics and chemical preparations on them. From a series of heterocyclic drugs, a drug with a wide spectrum of action was chosen as derivatives of benzimidazole and a model of a free-living unicellular eukaryote Endameba moshkovskii with a vegetative and cystic form.

The purpose of this work is the nature of the action of drugs of the benzimidazole series and in the ultrastructural visualization of the mechanism of action of benzimidazole using electron microscopic and electron-cytochemical methods on the model of polyxenic cultures of unicellular eukaryotes Entamoeba moshkovskii. We have established for the first time the excising effect of benzimidazole and the functional-ultrastructural mechanism of the action of benzimidazole on entameoba cells. As a result, the ultrastructural and functional morphology of benzimidazole action in the process of excysting entamoeba was established.

Keywords: Benzimidazole; Entamoeba; Cyst; Adenylate Cyclase

\section{Introduction}

It is known that various derivatives of heterocyclic compounds, including derivatives of benzimidazoles exhibit antimicrobial, protistocidal antifungal [1-5], cardioprotective, antitumor [6-9], activity and are used in practical medicine $[10,11]$. Among heterocyclic compounds of natural and synthetic origin, the benzimidazole ring is designated as a nucleus due to its presence in polyfunctional bioactive preparations [1-3], including vitamin B12 (Figure 1).

The use of plant growth regulators in agriculture, which include heterocyclic compounds (benzimidazole derivatives), which have a certain effect on unicellular organisms of freeliving protists living in soil and water bodies. To determine the mechanism of action of benzimidazole, a search is underway for more economical and at the same time adequate models. We have previously studied the ultrastructural characteristics of various prokaryotes and protists, as well as the mechanism of action of antibiotics and chemotherapy drugs on them $[4,13]$.

From a series of heterocyclic drugs, a drug with a broad spectrum of action and a model of a free-living unicellular eukaryote Entamoeba moshkovskii with vegetative and cystic forms were selected as derivatives of benzoimidazole. The aim of this work is to elucidate the nature of the action of drugs of the benzimidazole series and in ultrastructural visualization of the mechanism of action of benzimidazole using electron microscopic and cytochemical methods on 
the model of polyxenic cultures of single-celled eukaryotes Entamoeba moshkovskii. As a result, the ultrastructural and functional morphology of benzimidazole action in the process of excysting entamoeba was established.

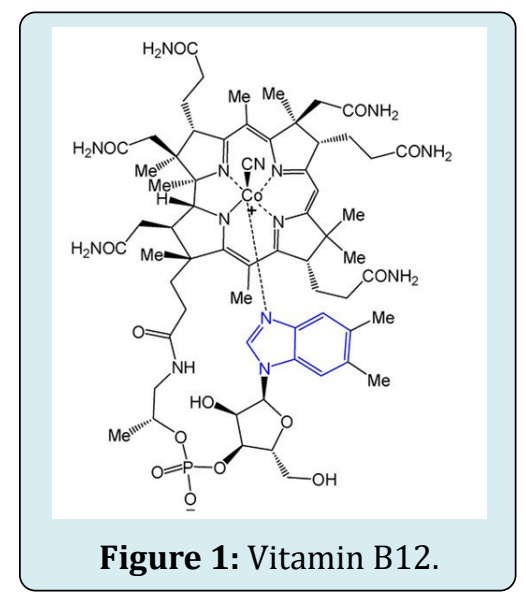

\section{Material and Methods}

\section{Experimental part}

The Entamoeba culture was used as a model of a singlecelled eukaryote. Moshkovskii Chalaya "Yer" isolated from urban wastewater [12]. During cultivation, we used Pavlov's single-phase medium at 250C. To determine the excising effect of various doses of benzimidazole, 10 samples of an 11-day-old culture of Entamoeba moshkovskii, where cystic forms of entamoeba prevailed, were used. The results of the study were evaluated in vitro under light and electron microscopy. For biometric measurements under a light microscope, an AT-9 oculare micrometer (MOV-15x) was used.

\section{Electron Microscopic and Cytochemical Parts}

Biological samples for electron microscopy were fixed with $2.5 \%$ glutaraldehyde in $0.1 \mathrm{M}$ cacodylate buffer at $\mathrm{pH} 7.4$ for 2hours. After washing three times in cacodylate buffer, postfixation was carried out with $1 \%$ osmium tetroxide in $0.1 \mathrm{M}$ cacodylate buffer at $\mathrm{pH} 7.4$ for 1 hour. After washing in the same buffer, biological samples were dehydrated in ethanol and acetone with an increasing concentration, then they were impregnated with a mixture of araldite [13]. After that, the samples were polymerized in a thermostat and ultrathin sections were obtained in an ultramicrotome (Reichert-Jung, Austria). Ultrathin sections were stained with $3 \%$ aqueous uranyl acetate and lead citrate. Microscopic examination was carried out on a Tesla-500 (Czech Republic) or JEOL-100 CX (Japan) transmission electron microscope (TEM). Determination of the localization of adenylae cyclase in entameb cells was performed according to the method proposed by Gayer G, et al. [14]; Reik J, et al. [15].

\section{Results and Discussion}

Our studies have shown that different concentrations of benzimidazole induce inappropriate cellular responses to Ent. moshkovskii. The benzylimidazole reaction is cytotoxic for entamoeba, which is manifested by immobilization of entameb and in the absence of pseudopodia. Determination of the amoebicidal concentration for an 11-day culture of $18 \mathrm{mg} / \mathrm{ml}$ Ent. moshkovskii showed where cystic resistant forms of entamoeba prevail Figure 2, Under the influence of benzimidazole at $4.7 \mathrm{mg} / \mathrm{ml}$ on an 11-day culture of Entamoeba, there is a massive excretion of entamoeba cysts with the formation of giant vegetative forms exceeding the usual vegetative cells in (Figure 3).

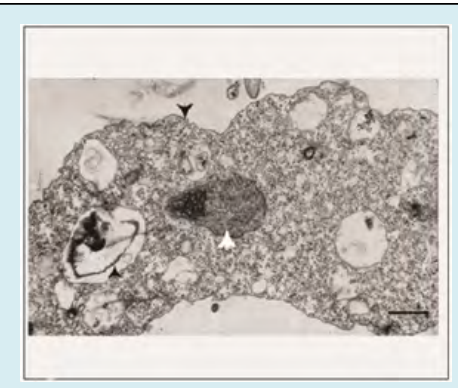

Figure 1: TEM. Vegetative form of Ent. moshkovskii. Scale bar: $1.0 \mu \mathrm{m}$.

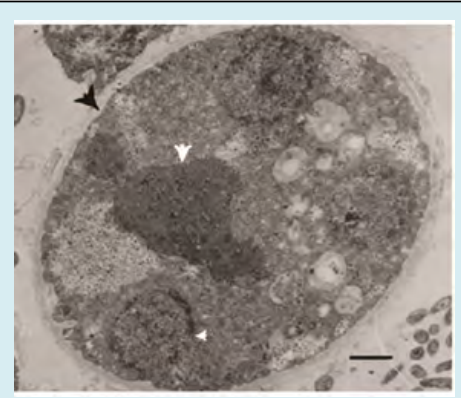

Figure 2: TEM. The cyst of Ent. moshkovskii. Scale bar: $1.0 \mu \mathrm{m}$.

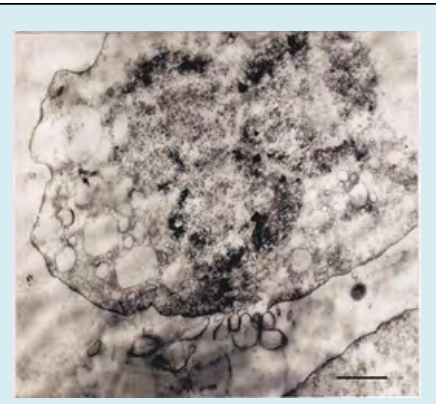

Figure 3: TEM. Excystation of Ent. moshkovskii. After effect of benzimidazole. Polynucleus cells vegetative form of Ent. moshkovskii. Scal bar: $1.0 \mu$. 


\section{Open Access Journal of Mycology \& Mycological Sciences}

The aim of this work is ultrastructural visualization using electron microscopic and cytochemical methods of the action of benzimidazole on the model of polyxenic cultures of the unicellular eukaryote Entamoeba moshkovskii.

Earlier, in the search for effective anti-amebic drugs, we studied the comparative protistocidal action of etiotropic, as well as sought-after anticancer drugs from the group of imidazoles, on entamoeba (Tables 1 \& 2).

\begin{tabular}{|c|c|c|c|}
\hline $\begin{array}{c}\text { Appellation } \\
\text { preparations }\end{array}$ & Entamoeba & moshkovskii & t \\
\hline Metronidazol & $0.6 \pm 0.03$ & $9.02 \pm 0.01$ & 10.12 \\
\hline
\end{tabular}

Table 1: Example t indicator explained in composition with the action of metronidazole on Ent. moshkovskii: $9.02 \pm 0.01$; $\mathrm{t}-10,12$.

Note: $t$ index is calculated in comparison with the effect of metronidazole on Ent. moshkovskii.

\begin{tabular}{|c|c|c|}
\hline Name of Preparates & Solution & Name of the drug Solvent Minimum inhibitory Concentration \\
\hline F-5469 & Water &.$>100$ \\
\hline CL-5650 & Dimethyl Sulfoxside & 75 \\
\hline Br-5651 & Dimethyl Sulfoxside & 75 \\
\hline 4-NO2.5658 & Dimethyl Sulfoxside & 41.5 \\
\hline 3-NO2.5653 & Water &.$>100$ \\
\hline NH2 & Water &.$>100$ \\
\hline
\end{tabular}

Table 2: The action of new drugs from the imidazole group on Ent. Moshkovskii.

The aim of this work is ultrastructural visualization using electron microscopic and cytochemical methods of the action of benzimidazole on the model of polyxenic cultures of the unicellular eukaryote Entamoeba moshkovskii.

According to some authors, the mechanism of benzimidazole action on eukaryotes is associated with a change in the permeability of the cytoplasmic membrane of cells, during which the proton pump is activated, which leads to an increase in cell volume [16-18]. On the one hand, benzimidazole affects the process of gene transcription and telomere preservation through an indirect effect on telomerase [19]. On the other hand, benzimidazole acts on the activation of adenylate cyclase, the location of which has been identified in the plasma membrane of entamoeba (Figures 4a-c).

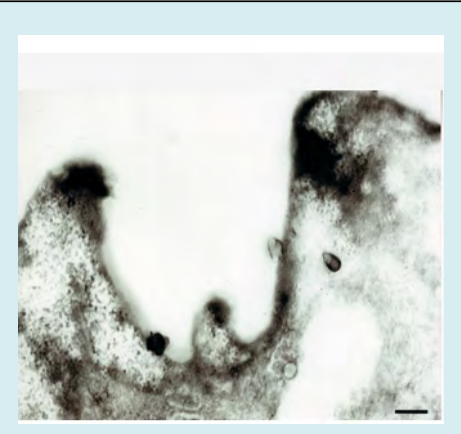

Figure 4a: TEM. Citochemistry reaction of adenilatcyclasa to plasmatic membrane of cell Ent. moshkovskii. Scale bar: $0.5 \mu \mathrm{m}$.
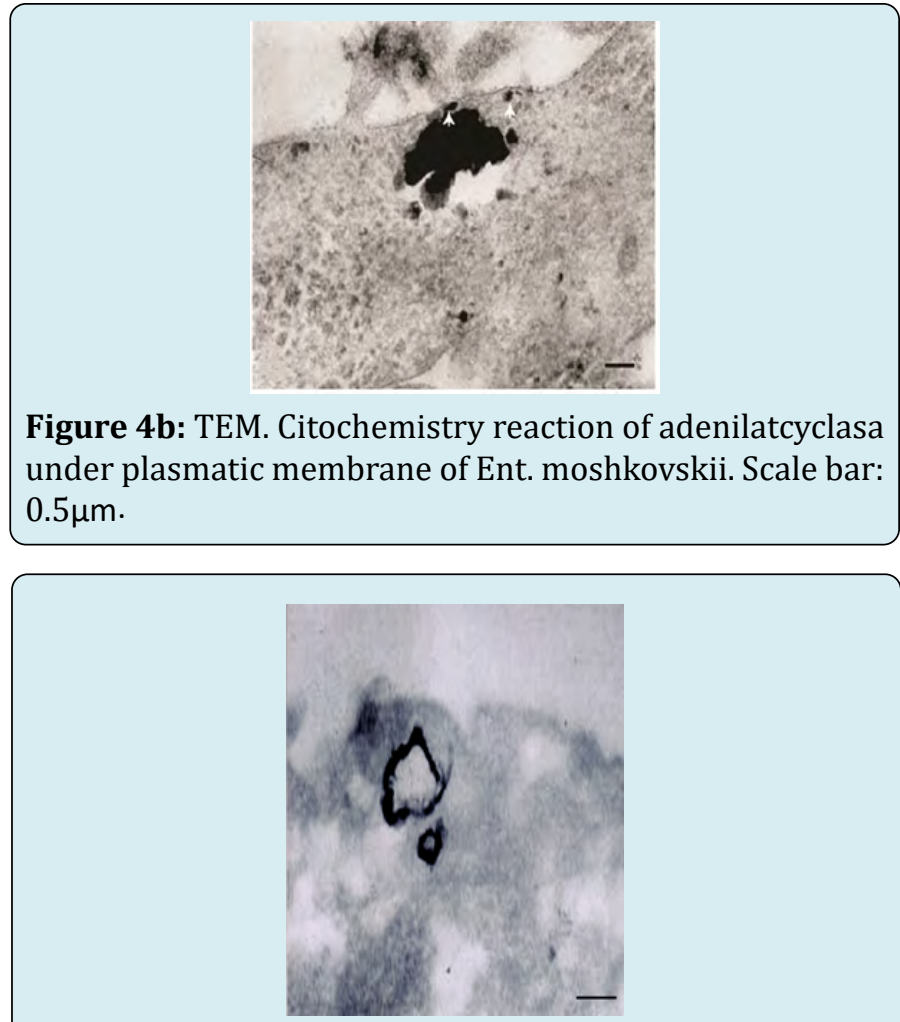

Figure 4C: TEM. Reaction of adenilatcyclase in the citoplasmatic vacuole of Ent. moshkovskii. Scale bar: $0.5 \mu \mathrm{m}$.

In our opinion, the molecular mechanism of benzimidazole action through the activation of adenyl cyclase, the detection of an electron- dense sediment under 


\section{Open Access Journal of Mycology \& Mycological Sciences}

the plasma membrane and in the cytoplasmic vacuole, identified using a cytochemical reaction, indicates its role in cell excretion.

\section{Conclusion}

Studies have shown the mechanism of the excystic action of low concentrations of benzimidazole on cysts of an 11-day culture of Ent. moshkovskii. Ultrastructural localization of adenylcyclase enzymatic activity in the plasma membrane of the entamoeba cell has been established. Taking into account the obtained data, it seems promising to continue a comparative study of the properties of etiotropic and antitumor drugs on simple economical models of unicellular eukaryotic cells.

\section{References}

1. Emerson G, Brink NG, Holly FW, Koniuszy F, Heyl D, et al. (1950) Vitamin B112-Like activity of 5, 6-dimethylbenzimidazole and tests on related compounds. J Am Chem Soc 72(7): 3084-3085.

2. Arutyunyan AA, Avakimyan JA, Stepanyan GM (2016) Antibacterial properties of some polycyclic heterocycles based on pyrimidine and benzimidazole. Biological J Armenia 68(2): 88-91.

3. Lubimova LK, Hovnanyan KO, Ivanova LN (1985) Contraindications to the use of polyethylene antibiotics. Antibiotici 3: 179-182.

4. Hovnanyan KO, Lubimova LK, Ivanova LN, Suhareva Nemakova LN (1987) Unstructural analysis of the left ventricle of Entamoeba. Antibiotici 12: 903-906. .

5. Iradyan MA, Iradyan NS, Stepanyan GM (2015) Expression of new antifungal preparations on the basis of pesticides. CSTOPHCH, Yerevan 311-324. .

6. Algul O, Karabulut A, Canacankatan N, Gorur A, Sucu $N$ (2013) Apoptotic and anti-angiogenic effects of benzimidazole compounds: Relationship with oxidative stress mediated ischemia/reperfusion injury in rat hind limb. Antiinflamm Antiallergy Agents Med Chem 11(3): 267-275.

7. Yadav G, Ganguly S (2015) Structure activity relationship (SAR) study of benzimidazole scaffold for different biological activities: A mini-review. Eur J Med Chem 97:
419-443.

8. Narasimhan B, Sharma D, Kumar P (2012) Benzimidazole: A medicinally important heterocyclic moiety. Med Chem Res 21: 269-283.

9. Bansal Y, Silakari O (2012) The therapeutic journey of benzimidazoles: A review. Bioorg Med Chem 20(21): 6208-6236.

10. Rathod CP, Rajurkar R, Thonte S (2013) Benzimidazole synthesis and biological evaluation: A review Indo Am J Pharm Res 3: 2323-2329.

11. Paul AT (1998) Green Chemistry: Theory and Practice. Oxford University Press: UK.

12. Chalaya LE (1962) Materials for the study of Ent. moshkovskii. Honey. Parasitology and parasitic diseases 31(6): 701-702.

13. Hovnanyan KO, Davtyan HH, Sargsyan CA, Trchounian AA (2010) Nanostructures of some viruses, bacterial and protozoa cells: electron microscopy and morphometrical analysis. Reports NAS RA 110: 277-284

14. Gayer G (1974) Electronic histochemistry, pp: 488.

15. Reik J, Petzold GL, Higgins JA, Greengard P, Barnett RJ (1970) Electron microscopic localization adenylatciclase in cells.

16. Bobik K, Boutry M, Duby G (2010) Activation of the plasma membrane $\mathrm{H}+$-ATPase by acid stress: antibodies as a tool to follow the phosphorylation status of the penultimate activating Thr. Plant Signal Behav 5: 681683.

17. Haruta M, Gray WM, Michael RS (2015) Regulation of the plasma membrane proton pump (H+-ATPase) by phosphorylation. Curr Opin Plant Biol 28: 68-75.

18. Haruta M, Burch HL, Nelson RB, Barrett-Wilt G, Kline $\mathrm{KG}$, et al. (2010) Molecular characterization of mutant Arabidopsis plants with reduced plasma membrane proton pump activity. J Biol Chem 285(23): 1791817929.

19. Zvereva ME, Shcherbakova DM, Dentsova OA (2010) Telomerase, structure, function and activity regulation. BioChemistry (Mosc) 75(13): 1563-1583. 